\title{
Ein einfaches Verfahren zur Ueberführung der Amidofettsäuren in die entsprechenden Monochlorfettsäuren.
}

\author{
Von
}

Apoth. Emil Jochem.

(Aus dem physiologisch-chemischen Institut zu Strassburg. Neue Folge Nr. 39.)

(Der Redaction zugegangen am 28. September 1900.)

Bei auf Anregung von Herrn Professor Hofmeister angestellten Versuchen aus den Spaltungsprodukten des Eiweisses, wie sie beim Zerkochen von Eieralbumin mit Salzsäure erhalten werden, durch Einwirkung von salpetriger Säure zu den zugehörigen Oxysäuren zu gelangen, erhielt ich vorwiegend gechlorte Verbindungen. Die ausgeätherten Reactionsprodukte hinterliessen nach dem Abdestilliren des Aethers einen Syrup, aus dem nach zweitägigem Stehen schöne, derbe Krystalle anschossen, die Chlor enthielten. Die Elementaranalyse ergab die für Chlorbernsteinsäure verlangten Zahlen.

Da Asparaginsäure ein constantes, wenn auch meistens nur in geringer Menge auftretendes Spaltungsprodukt der Eiweisskörper darstellt, lag die Vermuthung nahe, dass die gefundene Chlorbernsteinsäure von dieser abstamme, was mich veranlasste, zu untersuchen, wie sich unter den gewählten Reactionsbedingungen die Asparaginsäure verhalten möge. Auch in diesem Falle erhielt ich dieselbe Säure.

Es wurde nun mit wechselnden Mengen von Salzsäure und Nitrit der Versuch angestellt, aus verschiedenen Aminosäuren und Basen zu chlorirten Produkten zu gelangen. Die beste Ausbeute erhielt ich beim Lösen bezw. Suspendiren der Aminosäuren in der zehnfachen Menge concentrirter Salzsäure und tropfenweisem Zufügen von molekularen Mengen Natriumnitrit 
in concentrirter Lösung. Das Resultat fiel positiv aus für die Aminosäuren der Essig- und Oxalsäurereihe, ferner für die Aminosäuren der aromatischen Reihe mit dem Sitze des $\mathrm{NH}_{2}$ in der Seitenkette. Abnorm verlief die Reaction beim Tyrosin, wo anscheinend neben der Chlorirung ein Ersatz der Hydroxylgruppe durch eine $\mathrm{NO}_{2}$-Gruppe vor sich geht. Die aromatischen Aminosäuren mit $\mathrm{NH}_{2}$ im Kerne, sowohl wie die aromatischen und aliphatischen Basen lassen sich nicht nach demselben Schema in chlorirte Produkte überführen, wenigstens gelang es mir nicht, solche zu erhalten.

Diese Befunde schliessen sich den Beobachtungen von Curtius ${ }^{1}$ ) und von Tilden ${ }^{2}$ ) an, nur dass sich bei dem von mir angewandten Verfahren die gechlorten Produkte unter besonders leicht herstellbaren Bedingungen erhalten lassen.

Curtius brachte aus $\boldsymbol{\alpha}$-Aminofettsäuren dargestellte Diazofettsäureester mit concentrirter Salzsäure in Contact und bemerkte, dass sämmtlicher Stickstoff entwich und ein s substituirtes Produkt zurückblieb.

Direkten Ersatz der Aminogruppen durch Chlor glaubte Tilden durch Einwirkung von Nitrosylchlorid auf Amine erzielen zu können. Er erhielt aus Glycocoll Monochloressigsäure und aus Asparagin Chlorbernsteinsäure. Man darf wohl die Einwirkung von Nitrosylchlorid, die sich in erwähntem Sinne erst bei höherer Temperatur geltend macht, jener des Gemenges von salpetriger Säure und Salzsäure gleichstellen und somit auf die Reaction von Curtius zurückführen.

Vor dem Verfahren von Curtius hat mein Vorgehen voraus, dass es die Darstellung des Aminosäureesters umgeht und ohne vorgängige Isolirung der Diazoverbindungen zum Ziele führt. Von besonderem Nutzen dürfte diese Art der Gewinnung von chlorirten Säuren sein in den allerdings seltenen Fällen, wo die Aminosäuren leichter zu beschaffen sind als die entsprechenden freien Säuren, so beim Leucin und der Glutamin-

1) Curtius, B. B. Bd. 16, S. 754, 1883.

2) Tilden, Journ. chem. Soc., 1895, I, 489 B. B. Bd. 28, Ref. S. 646, 1895; Ch. Cbl., 1895, II., 30. 
säure, eventuell auch für die Darstellung der activen Modificationen von gechlorten Säuren aus den in der Natur vorkommenden activen Aminosäuren. Da die Reaction schon bei Zimmertemperatur vor sich geht, dürften einheitliche, optisch active Produkte entstehen.

Ein unter Umständen werthvoller Vorzug dieses Verfahrens von Chloreinführung anderen Methoden der Chlorirung gegenüber liegt darin, dass unter den von mir eingehaltenen Bedingungen der Eintritt des Chlors auf den Ort der $\mathrm{NH}_{2}$ Gruppe beschränkt bleibt. Auch für den qualitativen Nachweis von Aminofettsäuren kann die Reaction dienstbar gemacht werden. Man löst oder suspendirt die betreffende Substanz in der zehnfachen Menge concentrirter Salzsäure, behandelt mit Natriumnitritlösung, wobei das gechlorte, mit Aether extrahirbare Produkt entsteht. Der Verdunstungsrückstand des Aethers wird mit Salpetersäure angesäuert und mit Silbernitratlösung im Ueberschuss versetzt, wobei anhaftende Salzsäure niedergeschlagen wird. Das Filtrat liefert mit concentrirter Salpetersäure gekocht von Neuem einen reichlichen Chlorsilberniederschlag. Die Entstehung von chlorsubstituirten Fettsäuren vom Glycocoll aufwärts macht sich überdies schon durch das Auftreten öliger Tropfen bemerkbar.

Für die physiologische Chemie dürfte die Reactionsfähigkeit der Aminosäuren nach dieser Richtung hin insofern von Interesse sein, als man damit Aufschluss über die das Eiweissmolekül bildenden Mono- und Diaminosäuren in anderer als bisher eingeschlagener Richtung erhalten kann. Herr Professor Hofmeister hat für die Weiterführung der Untersuchungen unter Zugrundelegung dieser Erfahrungen bereits Sorge getragen.

Im Nachstehenden theile ich einige einschlägige Versuche mit, welche über die Anwendbarkeit des Verfahrens zur Darstellung bekannter und Auffindung neuer Chlorfettsäuren ein Urtheil ermöglichen.

\section{Darstellung von Chlorbernsteinsäure aus Eieralbumin.}

Ich kochte Eiereiweiss mit der dreifachen Menge concentrirter Salzsäure auf dem Sandbade während fünf Stunden 
am Steigrohre, filtrirte nach dem völligen Erkalten die schwarzbraune Lösung von ausgeschiedenen schwarzen Produkten, «Melaninen », ab und liess unter Umrühren und Kühlen mit Wasser eine gesättigte wässerige Lösung von Natriumnitrit aus einer Pipette zufliessen. Die Aminosäuren mögen hier in etwa $20 \%$ iger Salzsäure gelöst gewesen sein. Die angewandte Menge salpetrigsaures Natrium betrug $80 \mathrm{~g}$ auf $100 \mathrm{~g}$ Eiweiss, $10 \%$ mehr als die auf $15 \%$ Stickstoff berechnete Menge. Nachdem etwa die Hälfte der Natriumnitritlösung verbraucht war, nahm die Flüssigkeit eine hellgelbe Färbung an und trübte sich unter Ausscheidung öliger Tropfen, die sich auf dem Boden des Gefässes ansammelten. Auf der Oberfläche schwamm eine schwarze harzige Masse, die entfernt wurde. Es trat reichliche Entwicklung von Stickstoff und Kohlensäure auf. Die zweite Hälfte der Natriumnitritlösung wurde ebenfalls vorsichtig zugefügt und das Gemisch alsdann eine Zeit lang sich selbst überlassen. Um die überschüssige salpetrige Säure und das Stickoxyd zu vertreiben, leitete ich eine Stunde lang Luft durch das Gemenge und trennte die beiden Schichten im Scheidetrichter. Die hellgelbe wässerige Lösung wurde durch Schütteln mit Aether erschöpft und die ätherische Lösung auf einem mässig erwärmten Wasserbade abdestillirt. Aus dem syrupösen Rückstand krystallisirte ein Körper aus, der von der Mutterlauge abgesaugt, mit wenig Aether nachgewaschen und durch Umkrystallisiren aus letzterem Lösungsmittel gereinigt wurde. Die Krystalle zeigten den Schmelzpunkt $174^{\circ}$.

Die im Vacuum-Exsiccator getrocknete Säure ergab folgende Werthe:
Berechnet für $\mathrm{C}_{4} \mathrm{H}_{5} \mathrm{ClO}_{4}$ :
C. $31,48 \%$
I.
II.
H $\quad 3,27 \%$ $31,61 \%$ $31,71 \%$
Cl1) $23,25 \%$
$3,45 \%$
$3,39 \%$
$23,02 \% \quad 23,13 \%$.

Es lag sonach eine Monochlorbernsteinsäure vor, welche

1) Obige Chlorbestimmungen sowohl als die folgenden wurden nach Carius ausgeführt, die Verbrennungen durch Mischen der Substanzen mit Bleichromat. 
ihrem Schmelzpunkte nach mit einer der activen Modificationen, vermuthlich mit der von Tilden und Marshall aus Asparagin durch Nitrosylchlorid neben Fumarsäure erhaltenen l-Chlorbernsteinsäure identisch ist.

\section{Darstellung von Monochloressigsäure auf Glycocoll.}

$5 \mathrm{~g}$ käufliches Glycocoll wurden mit der zehnfachen Menge concentrirter Salzsäure angerieben und unter Kühlen mit einer gesättigten Natriumnitritlösung, enthaltend $5 \mathrm{~g}$ salpetrigsaures Natrium, tropfenweise versetzt; dann überliess ich das Gemisch einige Zeit sich selbst, leitete Luft durch und ätherte aus. Der ätherische Rückstand krystallisirte im Exsiccator aus und wog 2,3 g. Da Monochloressigsäure in Wasser leichter löslich ist als in Aether, kann durch Ausäthern nicht festgestellt werden, ob die Reaction quantitativ verläuft, doch kann man mit Sicherheit annehmen, dass $40 \%$ des angewendeten Glycocolls als gechlorte Säure erhalten werden.

Die durch Destillation rein erhaltenen Krystalle schmolzen bei $62^{\circ}$.

Berechnet für $\mathrm{C}_{2} \mathrm{H}_{3} \mathrm{ClO}_{2}$ :

Gefunden:
Cl $33,48 \%$.
I.
$33,59 \%$
II.
$33,53 \%$

Darstellung von Phenylchloressigsäure aus Phenylaminoessigsäure.

Die Aminosäure wurde nach der Methode von Tiemann ${ }^{1}$ ) dargestellt. $10 \mathrm{~g}$ des Präparates wurden genau wie bei den vorhergehenden Aminosäuren in salzsaurer Lösung mit $5,5 \mathrm{~g}$ Natriumnitrit behandelt, das Reactionsprodukt ausgeäthert und der Destillationsrückstand, ein gelber Syrup, zur Krystallisation in den Exsiccator gestellt. Die Ausbeute an Rohprodukt betrug $8,3 \mathrm{~g}$, war also nahezu quantitativ. Durch Umkrystallisiren aus heissem Ligroin erhielt ich die bei $78^{\circ}$ schmelzende gechlorte Säure.

Berechnet für $\mathrm{C}_{8} \mathrm{H}_{7} \mathrm{ClO}_{2}$ :

Gefunden:
Cl $20,79 \%$.
$20,67 \%$
$20,93 \%$

II.

1) Tiemann, B. B. Bd. 13, S. 383, 1880. 


\section{Darstellung von $\alpha$-Chlorglutarsäure aus Glutaminsäure.}

Die Glutaminsäure gewann ich aus käuflichem Casein durch Kochen mit der dreifachen Menge concentrirter Salzsäure während 5 Stunden am Steigrohre. Der Zerkochungsflüssigkeit setzte ich nach vorausgegangener Verdünnung mit Wasser auf je 100 Theile Casein 10 Theile Phosphorwolframsäure in Lösung zu und liess im Eiskasten absitzen. Von dem schlammigen Niederschlage und aufschwimmenden Fette wurde abfiltrirt, das Filtrat zur Syrupdicke eingeengt, mit Salzsäuregas gesättigt und das Gemisch über zuletzt genannter Säure unter einer Glasglocke an einem kühlen Orte aufgestellt. Schon nach einigen Stunden schieden sich aus der hellbraunen Lösung glänzende Krystalle aus; nach achttägigem Stehen war der Inhalt des Glases zu einer salbenartigen Masse erstarrt. Der Krystallbrei wurde an einer guten Saugpumpe während 10 Stunden abgesaugt, die Krystalle auf poröse Thonplatten aufgestrichen, welche die noch anhaftende Mutterlauge rasch aufsaugten, sodass auf dem Thone eine verfilzte, fast völlig weisse Kruste zurückblieb. Letztere wurde abgekratzt, in wenig Wasser gelöst, mit Thierkohle entfärbt und aus der farblosen Lösung die Glutaminsäure als salzsaures Salz durch Einleiten von gasförmiger Salzsäure ausgeschieden. 2 Kilo des noch stark verunreinigten technischen Caseins lieferten auf diese Weise behandelt $320 \mathrm{~g}$ reine salzsaure Glutaminsäure, die ich in Portionen zu $100 \mathrm{~g}$ auf $\omega$-Chlorglutarsäure verarbeitete.

Auf 100 Theile salzsaure Glutaminsäure verwandte ich 45 Theile Natriumnitrit und verfuhr genau wie vorher bei der Darstellung der gechlorten Produkte, nur wurde der ätherische Auszug diesmal nicht durch Destillation auf dem Wasserbade eingeengt, weil man sonst Gefahr läuft, dass sich die neue Säure zersetzt. Das Verjagen des Aethers geschah im Vacuum bei einer Temperatur, die $30^{\circ}$ nicht überschritt. Der Rückstand, ein gelber Syrup, wurde in eine flache Krystallisirschaale gebracht, durch Evacuiren im Exsiccator von dem noch anhaftenden Aether befreit, wobei sich die gechlorte Säure mikrokrystallinisch ausschied. Beim Aufstreichen auf Thonplatten 
verschwand die anhaftende Mutterlauge, und ein weisser Krystallüberzug blieb zurück. Nach öfterem Umkrystallisiren aus wasserfreiem Aether erhielt ich durch Ueberschichten der ätherischen Lösung mit Ligroin bis zur beginnenden Trübung grosse, einen Centimeter lange, pyramidenartige Gebilde, die aus lauter kleinen Krystallen aufgebaut waren. Die einzelnen Kryställchen waren jedoch zu klein, um einer Messung unterzogen werden zu können. Die Ausbeute überstieg nicht $20 \%$ der verarbeiteten salzsauren Glutaminsäure; ein Theil davon scheint durch Oxydation verloren zu gehen, denn es tritt neben Stickstoff auch reichliche Kohlensäureentwicklung auf.

Die Krystalle sinterten bei $97^{\circ}$ und waren bei $100^{\circ}$ ganz geschmolzen. Die ${ }_{a}$-Chlorglutarsäure löst sich sehr leicht in Wasser, Alkohol, Aether und Aceton, ist unlöslich in Benzol, Chloroform nnd Ligroin. Mit Wasser zersetzt sie sich aber leicht unter Abgabe ihres Chlors, das als Salzsäure in der Flüssigkeit gefunden wird; ebenso leicht zersetzlich sind die Salze in wässeriger Lösung. Die Analyse der bei $35^{\circ}$ getrockneten Substanz gab die für Monochlorglutarsäure erwarteten Werthe:

Berechnet für $\mathrm{C}_{5} \mathrm{H}_{7} \mathrm{ClO}_{4}$ :

$\begin{array}{cc} & \\ \text { I. Gefunden: } & \text { II. } \\ 36,24 \% & 36,16 \% \\ 4,30 \% & 4,29 \% \\ 21,42 \% & 21,38 \% .\end{array}$

\section{Baryumsalz.}

Etwa $2 \mathrm{~g}$ reiner Säure wurden in $20 \mathrm{~g}$ absolutem Methylalkohol gelöst und mit einer methylalkoholischen Barytlösung neutralisirt. Es schied sich sofort das Baryumsalz als weisser, voluminöser Niederschlag aus, der nach dem Auswaschen mit Methylalkohol und Trocknen bei $35^{\circ}$ zur Baryum- und Chlorbestimmung diente. Das Salz ist in Wasser leicht löslich, aber nicht ohne Chlorabspaltung, und fällt auf Zusatz von Alkohol wieder aus:

Berechnet für $\mathrm{C}_{5} \mathrm{H}_{5} \mathrm{ClO}_{4} \mathrm{Ba}$ :

Gefunden:

\begin{tabular}{|c|c|}
\hline $\mathrm{Cl} \quad 11,75 \%$ & $11,55 \%$ \\
\hline Ba $45,52 \%$ & $45,49 \%$ \\
\hline
\end{tabular}




\section{Kupfersalz.}

3,4 g reine Säure wurden in absolutem Methylalkohol gelöst, mit methylalkoholischem Ammoniak genau neutralisirt und mit einer Lösung von Kupferchlorid in genanntem Lösungsmittel ausgefällt. Der hellblaue Niederschlag wurde ausgewaschen und getrocknet. Die Versuche, sowohl das Kupfersalz als das Baryumsalz aus Wasser umzukrystallisiren, scheiterten an der leichten Zersetzlichkeit der Chlorglutarsäure.

$$
\text { Analyse: }
$$

Berechnet für $\mathrm{C}_{5} \mathrm{H}_{5} \mathrm{ClO}_{4} \mathrm{Cu}$ :

Gefunden:

$\begin{array}{lcc}\text { Cl } 15,55 \% & \text { I. } & \text { II. } \\ \mathrm{Cu} 27,99 \% & 15,53 \% & 15,37 \% \\ & 27,83 \% & 27,91 \% .\end{array}$

$\alpha$-Chlorglutarsäure-Diäthylester.

$5 \mathrm{~g}$ reiner Säure wurden in absolutem Aethylalkohol, der zuvor mit Salzsäuregas bei $0^{0}$ gesättigt war, gelöst, die Lösung während 3 Tagen im Eisschranke sich selbst überlassen, alsdann in Wasser gegossen, der Ester im Scheidetrichter abgetrennt und mit Natriumcarbonatlösung geschüttelt, bis die saure Reaction verschwunden war. Das anhaftende Natriumcarbonat lässt sich durch Ausschütteln mit Wasser, dem gegenüber der Ester weit beständiger ist als die Säure und ihre Salze, leicht auswaschen. Ich trocknete den Ester in ätherischer Lösung über Chlorcalcium und destillirte im Vacuum.

$\alpha$-Chlorglutarsäure-Diäthylester ist schwerer als Wasser, hat das specifische Gewicht 1,14 bei $23^{\circ}$, siedet im Vacuum (15 mm. Druck) bei $140-145^{\circ}$, bei gewöhnlichem Druck nicht unzersetzt etwa bei. $245^{\circ}$ (i. D.) und besitzt angenehmen Geruch.

Berechnet für $\mathrm{C}_{9} \mathrm{H}_{15} \mathrm{ClO}_{4}$ :

Gefunden:

$$
\begin{array}{cr}
\text { C } & 48,56 \% \\
\text { H } & 6,75 \% \\
\text { Cl } & 15,94 \%
\end{array}
$$

\begin{tabular}{rr}
\multicolumn{2}{c}{ Gefunden: } \\
I. & II. \\
$48,64 \%$ & $48,47 \%$ \\
$6,82 \%$ & $6,85 \%$ \\
$15,76 \%$ & $16,09 \%$.
\end{tabular}

Ueberführen der $\alpha$-Chlorglutarsäure in die entsprechende Oxysäure.

Wie bereits erwähnt, zersetzt sich die $\alpha$-Chlorglutarsäure leicht in Wasser, indem sie Chlor als Salzsäure abgibt; das 
entstehende Produkt musste also eine Oxysäure sein. Ich stellte eine $5 \%$ ige wässerige Lösung der Säure dar und liess dieselbe 2 Tage in einem Digestorium bei $40-50^{\circ}$ stehen, neutralisirte alsdann mit Natronlauge und dampfte auf ein kleines Volumen ein. Die mit Schwefelsäure angesäuerte Lösung wurde ausgeäthert und der Auszug auf dem Wasserbade abdestillirt. Der syrupöse Rückstand erstarrte über Schwefelsäure aufbewahrt zu einer krystallinischen Masse vom Schmelzpunkte $72^{\circ}$.

Um mich zu vergewissern, dass die Säure auch die verlangte $\alpha$-Oxysäure war, stellte ich das charakteristische Zinksalz dar. Die Krystallmasse wurde in wässeriger Lösung mit Zinkcarbonat erwärmt, das überschüssige kohlensaure Zink mit Wasser wiederholt ausgekocht und die vereinigten Filtrate eingedampft. Der hinterbliebene Syrup krystallisirte zum Theil über Nacht aus. Die Krystalle liessen sich mit wenig Wasser von der anhaftenden dickflüssigen Mutterlauge befreien. Tags darauf zeigte das Waschwasser eine reichliche Ausscheidung von Krystallen, welche abgesaugt und mit der ersten Portion zusammen durch Umkrystallisiren gereinigt wurden. Beim Lösen bedurfte es aber einer grösseren Menge Wassers als zuvor; schon beim Eindampfen schieden sich warzenförmige Krystalle aus, die nach dem Erkalten der Lösung sich noch vermehrten und mikroskopisch untersucht aus lauter kleinen Tafeln bestanden.

Die Zinkbestimmungen in dem bei $125^{\circ}$ getrockneten Salze ergaben:

Berechnet für $\mathrm{ZnC}_{5} \mathrm{H}_{8} \mathrm{O}_{5}$ :

Gefunden:

$$
\begin{array}{ccc} 
& \text { I. } & \text { II. } \\
\text { Zn } 30,94 \% & 30,86 \% & 31,09 \% .
\end{array}
$$

Es unterliegt also keinem Zweifel mehr, dass das aus Glutaminsäure erhaltene gechlorte Produkt die zugehörige $\alpha$-Chlorglutarsäure ist.

Darstellung von $\alpha$-Chlorisobutylessigsäure aus Leucin.

Das verarbeitete Leucin wurde zum grössten Theile erhalten durch 24stündiges Kochen von Hornspänen mit 
25\% iger Schwefelsäure. ${ }^{1}$ ) Die Zersetzungsflüssigkeit wurde mit gelöschtem Kalk vom grössten Theile der Schwefelsäure befreit und eingedampft. Die durch Stehenlassen aus der gelben Flüssigkeit erhaltenen Krystallmassen gaben in wässeriger Lösung mit Thierkohle gekocht ein farbloses Filtrat, das in der Siedehitze mit Kupfercarbonat behandelt, einen Niederschlag von Leucinkupfer bildete, welcher nach dem Auswaschen mit Schwefelwasserstoff zerlegt wurde. Das erhaltene Leucin krystallisirte ich aus ammoniakalischem Alkohol um und erhielt auf diese Weise ein von Tyrosin freies Präparat. Es gab wenigstens keine Millon'sche Reaction mehr.

Die Ausbeute an Leucin war eine sehr unbefriedigende, was daher rührt, dass Leucinkupfer bei Anwesenheit von Kupfersalzen anderer Aminosäuren weit löslicher ist als in reinem Zustande. Aus dem Kupferniederschlage erhielt ich auf diese Weise aus 3 Kilo Hornspänen nicht mehr als $50 \mathrm{~g}$ Leucin.

Ich verarbeitete dasselbe in Portionen von $25 \mathrm{~g}$ wie das Glycocoll. Man muss auch hier mit Wasser kühlen, damit die Temperatur nicht über Zimmerwärme steigt. Als die Hälfte der Natriumnitritlösung verbraucht war, trübte sich das Gemisch durch ausgeschiedene ölige Tropfen, die sich an der Oberfläche sammelten. Durch Ausäthern gewann ich diese ölige Schicht und destillirte bei einer $30^{\circ}$ nicht übersteigenden Temperatur den Aether im Vacuum ab. Die neue Säure blieb als ölige, mit Wasser nicht mischbare Flüssigkeit zurück.

Aus den Salzen des so erhaltenen Rohproduktes die reine Säure $\mathrm{zu}$ erhalten ist, ihrer leichten Zersetzlichkeit wegen, nicht möglich; auch durch Verseifen des Esters kann die freie Säure nicht erhalten werden, ohne dass gleichzeitig alles Chlor eliminirt wird. Um die anhaftende Salzsäure und salpetrige Säure einigermaassen $\mathrm{zu}$ entfernen, sowie beigemengten gelben Syrup $\mathrm{zu}$ beseitigen, schüttelte ich in ätherischer Lösung zweimal mit schwach alkalischem Wasser

1) Hammarsten, Lehrbuch der physiolog. Chemie S. 269. 
durch. Die ätherische Schicht wurde durch ein zuvor mit Aether benetztes Filter abfiltrirt, mit Chlorcalcium getrocknet und der Aether im Vacuum vertrieben. Der Rückstand war bedeutend weniger gelb gefärbt. Nach zweitägigem Stehen im Exsiccator über Aetzkali benützte ich einen Theil davon zur Chlorbestimmung.

Da keine Salze ohne Abspaltung von Chlor zu erlangen waren, stellte ich aus dem Reste den Aethylester dar. Die Ausbeute an gechlorter Säure überstieg nicht $20 \%$ des angewendeten Leucins.

Die erhaltene Säure stellt eine ölige, schwach gelbe Flüssigkeit dar, welche specifisch schwerer als Wasser und damit nicht mischbar ist. Mit Alkohol und Aether mischte sie sich in jedem Verhältnisse. Selbst beim Einstellen in eine Kältemischung zeigt sie keine Spur von Krystallisation. Sie gibt schon bei $40^{\circ}$ Salzsäure $a b$ und lässt sich weder im Vacuum noch bei gewöhnlichem Druck destilliren.

Bei der Chlorbestimmung fand ich:

I.

II.

III.

$23,75 \% \quad 23,93 \% \quad 23,80 \%$. Berechnet für $\mathrm{C}_{6} \mathrm{H}_{11} \mathrm{ClO}_{2} 23,56 \%$.

\section{Aethylester der $a$-Chlorisobutylessigsäure.}

8 Gramm des auf obige Art von Salzsäure und salpetriger Säure befreiten Chlorproduktes wurden in die zehnfache Menge von bei $0^{0}$ mit Salzsäuregas gesättigtem, absolutem Aethylalkohol eingetragen und 3 Tage lang im Eisschranke stehen gelassen. Dann wurde in Eiswasser gegossen, der ausgeschiedene Ester im Scheidetrichter abgetrennt und die ihm anhaftende Säure durch Natriumcarbonatlösung beseitigt. Nach dem Trocknen mit Chlorcalcium in ätherischer Lösung destillirte ich im Vacuum bei $15 \mathrm{~mm}$. Druck über, wobei der Ester zwischen 91-950 überging.

Der $\alpha$-Chlorisobutylessigsäure-Aethylester stellt eine farblose, angenehm nach Ananas riechende, mit Wasser nicht mischbare Flüssigkeit dar vom specifischen Gewicht 1,01 bei $23^{\circ}$. Er siedet unter Zersetzung bei $190^{\circ}$ (i. D.).

Für den zu erwartenden Ester berechnet sich die 
Formel $\mathrm{C}_{8} \mathrm{H}_{15} \mathrm{ClO}_{2}$, für welche Chlorbestimmungen und Elementaranalysen befriedigende Zahlen gaben.

Berechnet:

$\begin{array}{lr}\text { C } & 53,79 \% \\ \text { H } & 8,41 \% \\ \text { Cl } & 19,87 \%\end{array}$

Gefunden:

I.

$53,93 \%$

$8,56 \%$

$19,73 \%$
II. $53,66 \%$ $8,49 \%$ $19,83 \%$.

Ueberführen des $\alpha$-Chlorisobutylessigsäure-Esters in $\alpha$-0xyisobutylessigsäure.

Um von dem Ester zu der betreffenden Oxysäure zu gelangen, verseifte ich $4 \mathrm{~g}$ davon mit heiss gesättigtem Barytwasser, dem etwas Baryumcarbonat zugesetzt wurde, um beim Kochen das lästige Stossen zu verbindern. Nach zehnstündigem Erhitzen war die Verseifung beendigt, der Rückstand wurde -wiederholt mit Wasser ausgekocht, in der Lösung der überschüssige Baryt mit Kohlensäure gefällt und das erhaltene Baryumsalz in der Hitze genau mit der benöthigten Menge Zinksulfat umgesetzt. Durch wiederholtes Auskochen des entstandenen Niederschlages erhielt ich das schwerlösliche Zinksalz der Oxysäure, welches beim Umkrystallisiren und Einengen der Lösung sich in seidenglänzenden Schuppen abschied, die mikroskopisch betrachtet aus feinen Nadeln bestanden. Das Salz krystallisirte mit zwei Molekülen Krystallwasser.

Berechnet für $\left(\mathrm{C}_{8} \mathrm{H}_{11} \mathrm{O}_{3}\right)_{2} \mathrm{Zn}+2 \mathrm{H}_{2} \mathrm{O}$ :

Gefunden:

$\begin{array}{lrrr} & & \text { I. } & \text { II. } \\ \mathrm{Zn} & 17,99 \% & 17,84 \% & 17,81 \% \\ \mathrm{H}_{8} \mathrm{O} & 9,90 \% & 9,57 \% & 9,64 \%\end{array}$

Die aus dem Zinksalze nach Ansäuern mit Schwefelsäure und Ausäthern gewonnene freie Säure schmolz bei $67-70^{\circ}$. Selbst nach öfterem Umkrystallisiren des Salzes erlangte ich keine Säure mit schärferem Schmelzpunkte.

Nach Waage ${ }^{1}$ ) schmilzt die aus activem Leucin erhaltene Oxysäure bei $73^{\circ}$, nach Gmelin ${ }^{2}$ ) die aus einem von Hefe und Casein abstammenden Leucin dargestellte active Oxysäure

1) W a age, Ann., Bd. 118, S. 87, 1861.

2) Gmelin, Zeitschr. f. physiolog. Ch., Bd. XVIII, S. 31, 1894. 
bei $72,5^{\circ}$, die aus Hämoglobin erhaltene bei $67-70^{\circ}$. Die von mir erhaltene Säure stimmt in diesem Punkte mit der aus Hämoglobin dargestellten überein, doch weicht sie von dieser wieder durch den Wassergehalt des Zinksalzes ab, der bei Gmelin 1/2, bei mir 2 Moleküle betrug. Der Grund dieser Verschiedenheiten ist bisher nicht aufgeklärt, doch will ich bemerken, dass das von mir bei der Gewinnung der Oxysäure benützte Leucin nicht ausschliesslich aus Keratin stammte, sondern zu einem kleinen Theil durch Selbstverdauung von Pancreas erhalten war.

Es ist danach nicht ausgeschlossen, dass das von mir benützte Präparat noch ein Gemenge verschiedener Leucine darstellte und dass auch die beschriebenen Chlorprodukte nicht ganz einheitlicher Natur waren. Auch die Bezeichnung. $\alpha$-Chlorisobutylessigsäure möchte ich für so lange als eine vorläufige ansehen, als die Isomerie der aus verschiedenen Eiweisskörpern und nach verschiedenen Methoden erhältlichen Leucine nicht genügende Klärung erfahren hat. 